\title{
The political replacement effect in a kinetic model of social dynamics with phase transition
}

\author{
M. Dolfin* \\ Department of Engineering, University of Messina, Italy \\ *Email address for correspondence: marina.dolfin@unime.it \\ Communicated by Elena De Angelis \\ Received on 12 01, 2016. Accepted on 02 02, 2018.
}

\begin{abstract}
The political replacement effect is an interesting socio-political hypothesis introduced by Acemoglu and Robinson and statistically tested. It may determine, under some conditions, the phenomenon of innovation blocking, possibly leading to economic backwardness in a society. In a previous paper, we have introduced a kinetic model with stochastic evolutive game-type interactions, analyzing the relationship between the level of political competition in a society and the degree of economic liberalization. In the present paper we model we model the possibility of having a sort of phase transition occurring in the system when the phenomenon of blocking of the introduction of technological innovation, intended in a broad sense, appears. Crossing a critical point, the rules of interactions change by means of slightly different transition probabilities nevertheless determining very significant differences in the resulting long-term solutions.
\end{abstract}

Keywords: Kinetic models, social systems, social phase transition.

AMS subject classification: 34K60, 91B69, 91B15

\section{Introduction}

In the last years, a growing attention has been put on the complex interactions between politics and macroeconomics, giving rise, in particular, to the so called new political macroeconomics [1], mostly focusing on how politics affects economic outcomes. It is well known, in fact, that in real world political institutions a major role is played by the relationship between politics and macroeconomic policy. Our approach represents an attempt to translate into the mathematical framework of the kinetic theory applied to social dynamics ( [2], [3], [4], [5], [6], [7], [8], [9], [10], [11], [12], [13], [14], [15] and references therein) a model of the game-theoretic literature on macroeconomic policy [16] with the idea that this approach could give new insights in both fields.

As stressed by Drazen [1], if economy is the study of the optimal use of scarce resources [17], this implies that "once the optimal policy is found, it will be implemented". Drazen defines the policy maker, from this viepoint, as a social welfare maximizer. A well established field of research stems from the simple observation that actual and past policies, in many countries and situations, differed from this optimal policy. Acemoglu and Robinsons [16,18], but Drazen [1] also, propose many different historical and actual examples.

Following the phenomenology introduced in [16], we propose a kinetic model in which three groups interact, each of them differently characterized by two variables, representing significant political-economic determinants [8]. Using the kinetic approach with stochastic game-type interactions, we consider the evolution within each group as due to an internal to the group-type of interactions and a network-type of interactions. In the last one the dynamic of each group is influenced by the marginal first order moments of the distributions on the other groups. The novelty of the present paper, with respect to [8], is that we introduce here two different phases in our system. One phase is characterized by a welfaremaximizing policy maker [1], whilst in the other phase the policy maker is not welfare-maximizing. The phase transition is based on the behavior of the so called innovation function introduced in [8] on the basis of the phenomenology suggested in [16]; characterizes the rulers return value when technological 
innovation, intended in a broad sense, is introduced in the society. From now on the phase in which the policy maker is not welfare-maximizing is called blocking phase borrowing the term blocking by [16]. The blocking phase is characterized by the fact that the incumbent rulers, policy maker in this case, do not introduce technological innovations, intended in a broad sense, due to the fear to loose political power. This phenomenon has been called in [16] political replacement effect. This interesting phenomenon has been statistically tested in [19]. Next to the present one it is introduced a section describing the phenomenology, the mathematical model representing the phenomenology and some numerical results. A section of conclusions close the paper.

\section{A kinetic model of a society with phase transition.}

\subsection{Basic mathematical representation}

The phenomenological description of the socio-economic system introduced in this paper strictly resembles the one introduced in [8], except for the introduction of the phase transition. Then, we introduce it briefly, especially focusing on the two different phases.

The general socio-economic system is constituted by three subgroups: rulers, political competing group and citizens. We model two different political-economic determinants for each subgroup. Rulers' political power and propensity to innovate, citizens' wealth and political opinion on the government and finally wealth and political power of the political competing group. In a discrete state framework, the microstate of each group is mathematically represented as

$$
\left\{\left(u_{i}, \nu_{r}\right), \quad i=0, \ldots, I ; \quad r=0, \ldots, R\right\} .
$$

with

$$
D_{u}=\left\{u_{i}=\frac{i}{I}, i=0, \ldots, I\right\} \subset[0,1], D_{\nu}=\left\{\nu_{r}=\frac{r}{R}, r=0, \ldots, R\right\} \subset[0,1] .
$$

characterizing the domain of the microscopic variables $u$ and $\nu$, respectively. The introduced variables take different meanings for each subgroup of the general socio-economic system; i.e. they represent the determinants for each group, as above sketched. The meaning of the variables for each group will be better highlighted in the following subsection.

For each subgroup, a probability mass function is defined on the microstate

$$
f_{i r}^{s}(t)=f^{s}\left(t, u_{i}, \nu_{r}\right):[0,1] \times D_{u} \times D_{\nu} \rightarrow[0,1], \quad s=1,2,3,
$$

characterizing, on each subgroup (i.e. $\forall s=1,2,3$ ), the distribution of the socio-economic determinants related to the specific subgroup considered.

The first-order moments related to the marginal probabilities on each group are

$$
\begin{aligned}
& \mathbb{E}_{\nu}^{s}(t)=\sum_{i=0}^{I} \sum_{r=0}^{R} \nu_{r} f_{i r}^{s}(t), \\
& \mathbb{E}_{u}^{s}(t)=\sum_{r=0}^{R} \sum_{i=0}^{I} u_{i} f_{i r}^{s}(t),
\end{aligned}
$$

$s=1,2,3$.

Evolution equations read

$$
\frac{d}{d t} f_{i r}^{s}(t)=J_{s}\left[\mathbf{f}^{s}\right]+\mathcal{J}_{s}\left[\mathbf{f}^{j}\right]
$$

with the initial condition $\left(\mathbf{f}^{s}\right)_{0}=\mathbf{f}^{s}(0), s=1,2,3$ and $j \in\{0, \ldots, s-1, s+1, \ldots 3\}$ with $\mathbf{f}^{s}=\left\{f_{i r}^{s}\right\}, i=0, \ldots, I, r=0, \ldots, R$. The term $J_{s}\left[\mathbf{f}^{s}\right](s=1,2,3)$ accounts for the net flow due to internal to the group-type of interactions; it may depend on the probability mass function of the $s$-th 


\section{Dolfin}

group through its first moments. The term $\mathcal{J}_{s}\left[\mathbf{f}^{j}\right](s=1,2,3, \quad j \in\{1, \ldots, s-1, s+1, \ldots 3\})$ accounts for network-type of interactions.

With respect to the internal to the group-type of interactions the following stylized phenomenology is assumed for each subgroup. Rulers: their propensity to innovate is directly related to their political power. Citizens: their support to the rulers is directly related to their wealth. Political competing group: their political power is directly related to their wealth.

With respect to the network-type of interactions the following stylized phenomenology is assumed for each subgroup. The political power of the rulers is directly related to the citizens' political opinion on the government and inversely related to the political power of the competing group. The wealth of the citizens is directly related to the propensity to introduce innovation of the rulers. The wealth of the political competing group is directly related to the propensity to introduce innovation of the rulers. The above introduced phenomenology, stylized as much as possible to try to catch the general dynamical basic features, will be represented by means of specific transition probabilities. An intuitive graphical representation on the general dynamical interaction may be the following, where the minus signs and the red colors symbolically represent negative returns.

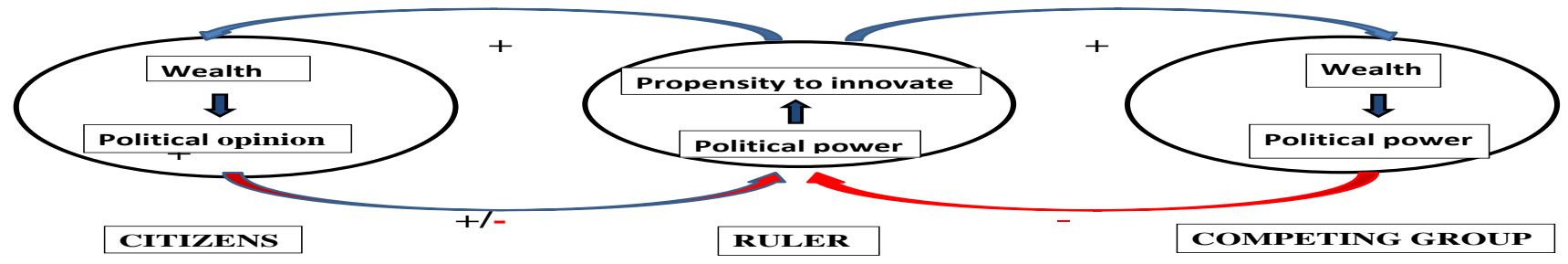

Figure 1. A sketch of the interactions between subgroups of the socio-economic system.

The explicit expressions for the flows of Equation (5), derived by balancing the inflow and the outflow of particles in an elementary volume of the microstates, are

$$
\begin{gathered}
J_{s}\left[\mathbf{f}^{s}\right]=\frac{1}{I+1} \sum_{h, k=0}^{I} \sum_{p, q=0}^{R} \eta_{s} \mathcal{D}_{h p}^{k q}(s)\left(\nu_{p} \rightarrow \nu_{r}\right) f_{h p}^{s} f_{k q}^{s}-f_{i r}^{s} \sum_{k=0}^{I} \sum_{q=0}^{R} \eta_{s} f_{k q}^{s}, \\
\mathcal{J}_{s}\left[\mathbf{f}^{j}\right]=\sum_{\substack{j=1 \\
j \neq s}}^{3}\left\{\frac{1}{R+1} \sum_{h=0}^{I} \sum_{p=0}^{R}\left[\mu_{s}^{j} \mathcal{B}_{h}^{j}(s)\left(u_{h} \rightarrow u_{i}\right) f_{h p}^{s}\right]-\mu_{s}^{j} f_{i r}^{s}\right\},
\end{gathered}
$$

for $s=1,2,3$, where $\eta_{s}$ is the frequency of internal to the group-type of interactions and $\mu_{s}^{j}$ is the frequency of network-type of interactions among the groups. Both of them are assumed to be constant in our model and they will be given as parameters of the model.

\subsection{Two different phases}

A blocking phase is characterized by rulers who are not welfare-maximizing due to the fear of losing their political power, more than their economic rents $[16,18]$; this effect may determines economic backwardness in the society, which affects the citizen wealth mean value. In particular, the condition of blocking occurs when the payoff of the rulers who do not innovate is bigger than their payoff when the innovation is introduced [16]. In [8] we analyzed the innovation function, which allows to quantify, in the 
kinetic mathematical framework, the blocking condition introduced in [16]

$$
\mathcal{F}\left(\mathbb{E}_{\nu}^{3}(t), \gamma ; \alpha\right)=\alpha P\left[\frac{1}{2}+\left(1-\mathbb{E}_{\nu}^{3}(t)\right)\right]-P\left[\frac{1}{2}+\gamma\left(1-\mathbb{E}_{\nu}^{3}(t)\right)-(\alpha-1)\right] .
$$

In (8) we used the following function

$$
P[h]=\left\{\begin{array}{lll}
0 & \text { if } & h<0 \\
h & \text { if } & 0 \leq h \leq 1 \\
1 & \text { if } & h>1
\end{array}\right.
$$

and in the following it will be clarified that the quantity $1-\mathbb{E}_{\nu}^{3}$ quantifies the inverse of the political competition; this quantity was introduced as a parameter in [16], whilst in our model depends on the dynamic of the whole system and changes with time.

The parameters [16] are:

- $\alpha$, which quantifies the effect of the introduction of technological innovation,

- $\gamma$ which quantifies the 'erosion' of the political power of the incumbent ruler due to the introduction of new technologies in the society,

The blocking condition is now derived as (for details see [8])

$$
\alpha P\left[\frac{1}{2}+\left(1-\mathbb{E}_{\nu}^{3}(t)\right)\right] \geq P\left[\frac{1}{2}+\gamma\left(1-\mathbb{E}_{\nu}^{3}(t)\right)-(\alpha-1)\right]
$$

The no blocking phase is characterized by welfare-maximizing rulers; the transition from the no blocking to the blocking phase is characterized by the behavior of the innovation function (8). When it is strictly less than zero, the blocking condition (9) applies, i.e. the payoff of the rulers who do not innovate is bigger than their payoff when the innovation is introduced, determining a behavior which is not welfare-maximizing.

In the no blocking phase the following transition probabilities are introduced for each group exactly as in [8]:

\section{Rulers.}

Microstate: $\left\{\begin{array}{lr}\text { political power } & \left(u_{i}, i=1, \ldots, I\right) \\ \text { propensity to innovate } & \left(\nu_{r}, r=0, \ldots, R\right)\end{array}\right.$

Internal to the group-type of interactions (in the no blocking phase):

The propensity to innovate of the rulers is directly related to their political power.

- if $\mathbb{E}_{u}^{1} \geq \frac{1}{2}$

$$
\forall k, q, \quad \mathcal{D}_{h p}^{k q}(1)\left(\nu_{p} \rightarrow \nu_{r}\right)=\left\{\begin{array}{lll}
0 & \text { for } & r \in\{0, \ldots, p-1\} \\
\frac{1}{R-p+1} & \text { for } \quad r \in\{p, \ldots, R\}
\end{array}\right.
$$

- if $\mathbb{E}_{u}^{1}<\frac{1}{2}$

$$
\forall k, q, \quad \mathcal{D}_{h p}^{k q}(1)\left(\nu_{p} \rightarrow \nu_{r}\right)=\left\{\begin{array}{lll}
\frac{1}{p} & \text { for } & r \in\{0, \ldots, p-1\} \\
0 & \text { for } & r \in\{p, \ldots, R\}
\end{array}\right.
$$

(in both cases above the transition probabilities are independent on the values of $\nu_{k}$ and $\nu_{q}$ ). 


\section{Dolfin}

Network-type of interactions:

The political opinion of the citizens on the rulers directly influences the political power of the rulers.

- if $\mathbb{E}_{\nu}^{2} \geq \frac{1}{2}$,

$$
\mathcal{B}_{h}^{2}(1)\left(u_{h} \rightarrow u_{i}\right)= \begin{cases}0 & \text { for } \quad i \in\{0, \ldots, h-1\} \\ \frac{1}{I-h+1} & \text { for } \quad i \in\{h, \ldots, I\}\end{cases}
$$

- if $\mathbb{E}_{\nu}^{2}<\frac{1}{2}$,

$$
\mathcal{B}_{h}^{2}(1)\left(u_{h} \rightarrow u_{i}\right)=\left\{\begin{array}{lll}
\frac{1}{h} & \text { for } & i \in\{0, \ldots, h-1\} \\
0 & \text { for } & i \in\{h, \ldots, I\} .
\end{array}\right.
$$

Finally, the political power of the competing group inversely influences the political power of the rulers.

- if $\mathbb{E}_{\nu}^{3} \geq \frac{1}{2}$,

$$
\mathcal{B}_{h}^{3}(1)\left(u_{h} \rightarrow u_{i}\right)=\tilde{\gamma} \delta_{i, h-1}+(1-\tilde{\gamma}) \delta_{i, h}
$$

- if $\mathbb{E}_{\nu}^{3}<\frac{1}{2}$,

$$
\mathcal{B}_{h}^{3}(1)\left(u_{h} \rightarrow u_{i}\right)=\delta_{h, i} .
$$

In the above equations and hereafter $\delta_{h, k}$ represents the Kronecker delta function and $\tilde{\gamma}$ quantifies the effect of the political power of the competing group on the political power of the rulers; it is the parameter $\gamma$ which has been normalized, i.e.

$$
\tilde{\gamma}=\frac{\gamma}{\gamma_{\max }},
$$

assuming that a maximal value exists for this quantity.

\section{Citizens.}

Microstate: $\begin{cases}\text { wealth } & \left(u_{i}, i=0, \ldots, I\right) \\ \text { political opinion } & \left(\nu_{r}, r=0, \ldots, R\right)\end{cases}$

internal to the group-type of interactions:

The opinion dynamics of the citizens is assumed to be driven simply by economic motivations: when two individuals interact, if the wealth status of the candidate particle is below the one of the field particle, the candidate particle has a probability to acquire the opinion of the field one, following an imitation rule. If, on the contrary, the wealth status of the candidate particle is greater or equal to the one of the field particle, the candidate one will not change his opinion. The following transition probabilities encode the above described phenomenology:

- if $u_{h}<u_{k}$,

$$
\mathcal{D}_{h p}^{k q}(2)\left(\nu_{p} \rightarrow \nu_{r}\right)=\beta \delta_{r, q}+(1-\beta) \delta_{r, p}
$$

- if $u_{h} \geq u_{k}$,

$$
\mathcal{D}_{h p}^{k q}(1)\left(\nu_{p} \rightarrow \nu_{r}\right)=\delta_{r, p}
$$

where $0 \leq \beta \leq 1$ is a parameter of the model and characterizes citizen susceptibility to change opinion. 
Network-type of interactions:

We model the impact of the introduction of technological innovations on the citizens income by simply reasoning that if the rulers introduce technological innovation, the citizens may, in probability, rise their wealth status.

- if $\mathbb{E}_{\nu}^{1} \geq \frac{1}{2}$,

$$
\mathcal{B}_{h}^{1}(2)\left(u_{h} \rightarrow u_{i}\right)=\tilde{\alpha} \delta_{i, h+1}+(1-\tilde{\alpha}) \delta_{i, h}
$$

- if $\mathbb{E}_{\nu}^{1}<\frac{1}{2}$,

$$
\mathcal{B}_{h}^{1}(2)\left(u_{h} \rightarrow u_{i}\right)=\delta_{h, i}
$$

where $\tilde{\alpha}$ quantifies the effect of the introduction of technological innovation by the rulers on the wealth of citizens; it is the parameter $\alpha$ which has been normalized, i.e.

$$
\tilde{\alpha}=\frac{\alpha}{\alpha_{\max }},
$$

assuming that a maximal value exists for this quantity.

\section{Competing group.}

Microstate: $\left\{\begin{array}{lr}\text { wealth } & \left(u_{i}, i=0, \ldots, I\right) \\ \text { political power } & \left(\nu_{r}, r=0, \ldots, R\right)\end{array}\right.$

Internal to the group-type of interactions:

in this case the political power of the competing group is simply assumed to be directly related to their wealth.

- if $\mathbb{E}_{u}^{3} \geq \frac{1}{2}$,

$$
\forall k, q, \quad \mathcal{D}_{h p}^{k q}(3)\left(\nu_{p} \rightarrow \nu_{r}\right)= \begin{cases}0 & \text { for } \quad r \in\{0, \ldots, p-1\} \\ \frac{1}{R-p+1} & \text { for } \quad r \in\{p, \ldots, R\}\end{cases}
$$

- if $\mathbb{E}_{u}^{3}<\frac{1}{2}$,

$$
\forall k, q, \quad \mathcal{D}_{h p}^{k q}(3)\left(\nu_{p} \rightarrow \nu_{r}\right)=\left\{\begin{array}{lll}
\frac{1}{p} & \text { for } & r \in\{0, \ldots, p-1\} \\
0 & \text { for } & r \in\{p, \ldots, R\}
\end{array}\right.
$$

(in both cases above the transition probabilities are independent on the values of $\nu_{k}$ and $\nu_{q}$ ).

Network-type of interactions:

We model the impact of the introduction of technological innovations on the competing group income by the simple reasoning that, as in the case of the citizens, if the rulers introduce technological innovation, the competing group may rise their wealth status.

- if $\mathbb{E}_{\nu}^{1} \geq \frac{1}{2}$

$$
\mathcal{B}_{h}^{1}(3)\left(u_{h} \rightarrow u_{i}\right)=\left\{\begin{array}{lll}
0 & \text { for } & i \in\{0, \ldots, h-1\} \\
\frac{1}{I-h+1} & \text { for } & i \in\{h, \ldots, I\}
\end{array}\right.
$$

- if $\mathbb{E}_{\nu}^{1}<\frac{1}{2}$

$$
\mathcal{B}_{h}^{1}(3)\left(u_{h} \rightarrow u_{i}\right)=\delta_{h, i}
$$




\section{Dolfin}

As a novelty with respect to [8], we introduce a new set of transition probabilities, i.e. new rules characterizing the interactions when the system enters the blocking phase. In the blocking phase, the only transition probability which is assumed to change is the internal to the group-type of interactions of the rulers, i.e.:

\section{Rulers.}

Internal to the group-type of interactions (in the blocking phase):

$$
\forall k, q, \quad \mathcal{D}_{h p}^{k q}(1)\left(\nu_{p} \rightarrow \nu_{r}\right)=\left\{\begin{array}{lll}
\frac{1}{p} & \text { for } & r \in\{0, \ldots, p-1\} \\
0 & \text { for } & r \in\{p, \ldots, R\},
\end{array}\right.
$$

The network-type of interactions of the rulers and all the transition probabilities of the other groups remain the (12)-(23).

\subsection{A case study: limited political competition}

An important conclusion of the model presented in the paper [16] is that political decision-makers are expected to support economic institutions in the case of strong autocracies with low level of political competition or in established democracies with high levels of political competition. On the other hand, it is when political competition is limited that development may be blocked by political elites. In the present case study we focus on this last case, i.e. we analyze the long term solutions of the system of equations (5) with the specifications (6), (7) and with initial conditions of limited political competition, explained in the following. The target of the numerical solutions which are presented is to show the effect of the 'phase transition' on the long term solutions, by comparing the solutions in condition of blocking (4) and without blocking (3). Moreover, another goal is to analyze the impact of a the parameter characterizing $\gamma$ which quantifies the erosion of the rulers' political power due to the introduction of technological innovation in the society. The $\gamma$ parameter appears worth to be analyzed for its socio-political implications, as documented also in [20]. To model the situation of a society with a limited political competition we assume as initial condition strong rulers (see Figure2 (left)) and a weak competing group (see Figure 2 (right)), whilst the wealth and political opinion of the citizens are uniformly distributed (see Figure 2 (center)). The same initial has been used in the first case study of paper [8], although we here compare the situation with and without phase transition. The initial conditions are represented using a bar graph representing the initial distribution for each group (parameter values are: $\alpha=1.1, \beta=0.3, \gamma=2$ ).
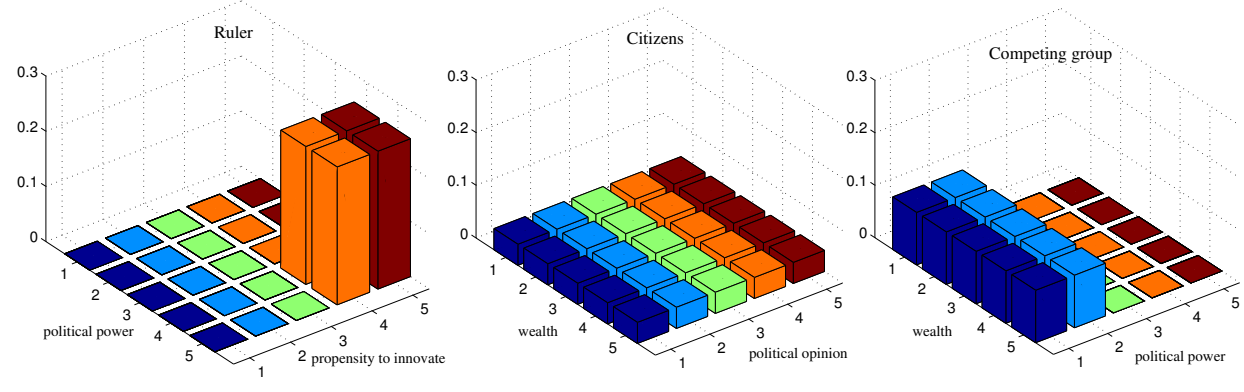

Figure 2. Bar graphs of the initial distribution for each group representing a society with limited political competition. 


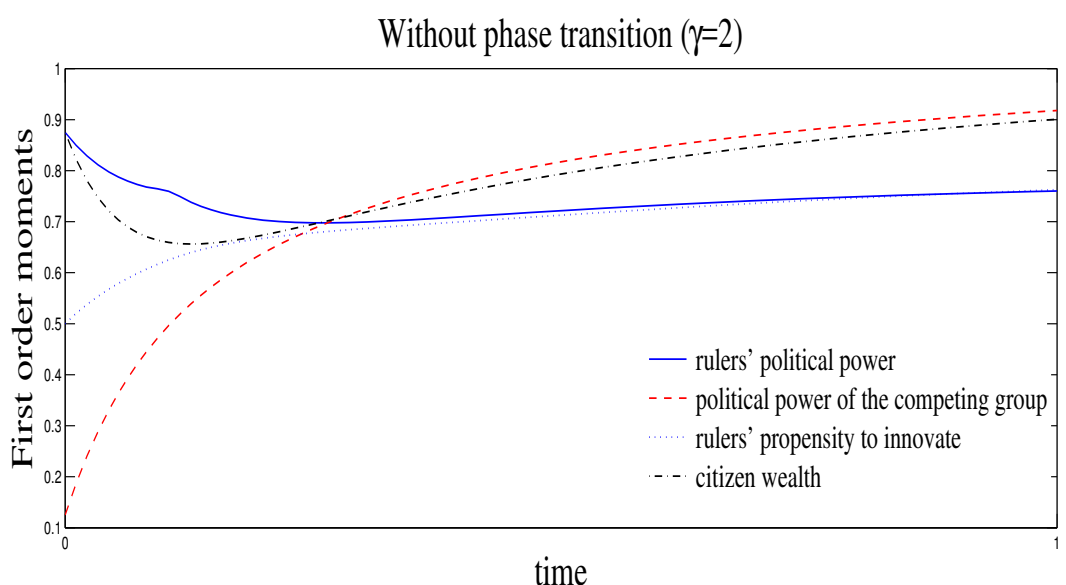

Figure 3. Time evolution of significant marginal first moments of the distributions in the three groups without phase transition.

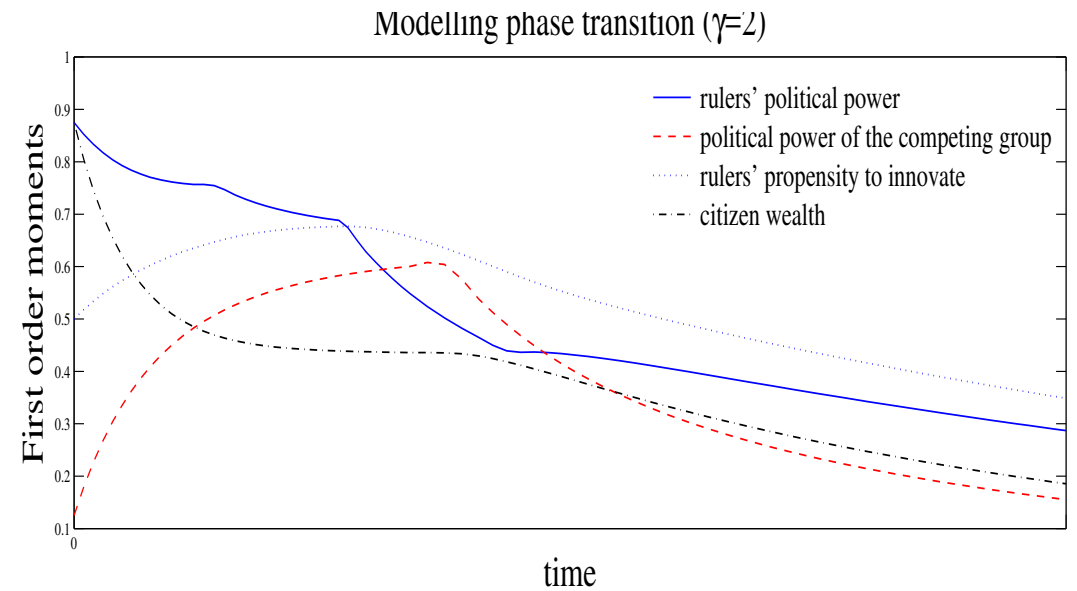

Figure 4. Time evolution of significant marginal first moments of the distributions in the three groups with phase transition.

The transition into the blocking phase determines a negative trend in the long term solution of the mean citizen wealth which was not observed when the solutions have been calculated in the same initial conditions and with the same value of the parameters but without introducing the possibility of having a phase transition. The transition to the blocking phase appears to be irreversible in this case. 


\section{Dolfin}

As mentioned in [16]: "A higher level of $\gamma$ increases the security of the ruler in the absence of innovation; hence it implies a greater erosion of entrenchment". To test the reliability of our kinetic model in reflecting the proposed socio-economic dynamics, we have numerically computed the same solution as the previous one, with the same initial conditions and parameters, except for the parameter $\gamma$ which takes the value $\gamma=10$ in the first solution and $\gamma=30$ in the second one.

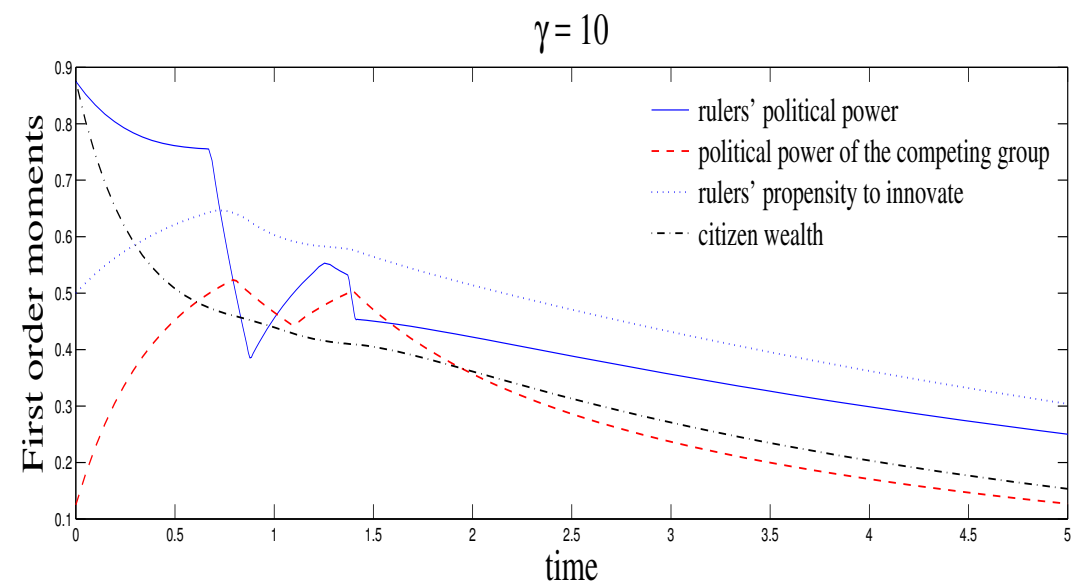

Figure 5. Time evolution with the same initial conditions and parameters as the previous one, except for $\gamma=10$.

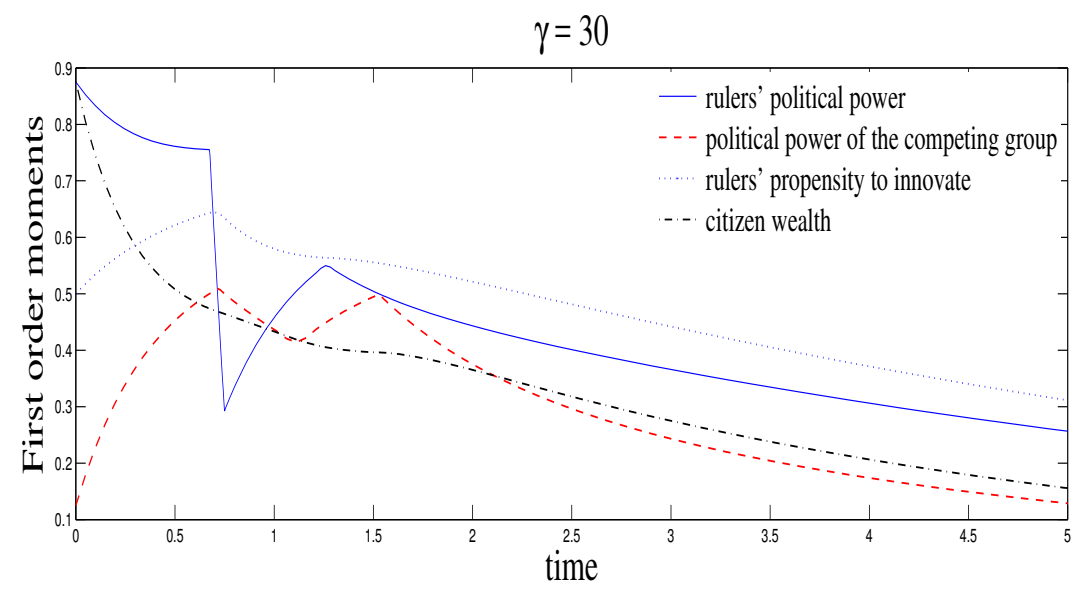

Figure 6. Time evolution with the same initial conditions and parameters as the previous one, except for $\gamma=30$.

The mean value of the political power of the rulers abruptly decreases more and more as $\gamma$ increases. Roughly speaking one may say that the transition into the blocking phase determines in any case a stabilization of all the mean values, although they are quite low. This kind of behavior may be considered as inefficient government from the political-economic viewpoint. It is evident that the hypothesis formulated in the context of this case study needs to be theoretically justified and numerically tested in more different case studies. 


\section{Conclusions}

In this paper we have proposed a kinetic model with stochastic game-type interactions representing a simple society with three interacting group of interests, each of them characterized by two politicaleconomic determinants. Two phases have been introduced in the society: a no blocking phase in which the government is welfare-maximizing and a blocking phase in which the government is not welfare-maximizing and may block the introduction of technical innovations in the society. The two different phases have been characterized by different transition probabilities and the phase transition has been identified by means of a blocking condition introduced in a model by Acemoglu [16]. Numerical results on a case study of a society with limited political competition are analyzed. The long term solutions of the model have been numerically evaluated in the condition of limited political competition. The numerical results have been obtained in two different cases; i.e. when the phase transition has not been taken into consideration and in the case in which it has been considered. The results about the time evolution of some significant first order moments of the marginal distributions of the different groups have been compared with those obtained with the same initial conditions and values of the parameters, but without introducing the event of the phase transition.

\section{References}

1. A. Drazen, Political Economy in Macroeconomics. Princeton University Press, 2001.

2. G. A. Marsan, N. Bellomo, and L. Gibelli, Stochastic evolving differential games toward a systems theory of behavioral social dynamics, Mathematical Models and Methods in Applied Science, vol. 26, p. 1051, 2016.

3. L. Arlotti, E. D. Angelis, L. Fermo, M. Lachowicz, and N. Bellomo, On a class of integro-differential equations modeling complex systems with nonlinear interactions, Applied Mathematics Letters, vol. 25, pp. 490-495, 2012.

4. N. Bellomo, D. Knopoff, and J. Soler, On the difficult interplay between life, "complexity", and mathematical sciences, Mathematical Models and Methods in Applied Science, vol. 13, pp. 1861-1913, 2013.

5. N. Bellomo, F. Colasuonno, D. Knopoff, and J. Soler, From systems theory of sociology to modeling the onset and evolution of criminality, Networks and Heterogeneous Media, vol. 10, pp. 421-441, 2015.

6. A. Bellouquid and E. D. Angelis, From kinetic models of multicellular growing systems to macroscopic biological tissue models, Nonlinear Analysis: Real World Applications, vol. 12, pp. 1111-1122, 2011.

7. M. Dolfin, L. Leonida, and N. Outada, Modeling human behavior in economics and social science, Physics of Life Reviews, vol. 22-23, pp. 1-21, 2017.

8. M. Dolfin, D. Knopoff, L. Leonida, and D. M. A. Patti, Escaping the trap of 'blocking': a kinetic model linking economic development and political competition, Kinetic and Related Models, vol. 10, pp. 423-443, 2017.

9. M. Dolfin and M. Lachowicz, Modeling altruism and selfishness in welfare dynamics: the role of nonlinear interactions, Mathematical Models and Methods in Applied Science, vol. 24, pp. 2361-2381, 2014.

10. M. Dolfin and M. Lachowicz, Modeling opinion dynamics: how the network enhances consensus, Networks and Heterogeneous Media, vol. 10, pp. 877-896, 2015.

11. M. Dolfin, From vehicle-driver behaviors to first order traffic flow macroscopic models, Applied Mathematics Letters, vol. 25, no. 12, pp. 2162-2167, 2012.

12. D. Bruneo, M.Dolfin, F. Longo, M. Scarpa, and A. Puliafito, Kaos: a kinetic theory tool for modeling complex social systems, MATEC Web of Conferences, vol. 68, p. 16004, 2016.

13. B. During and G. Toscani, International and domestic trading and wealth distribution, Communications in Mathematical Sciences, vol. 6, pp. 1043-1058, 2008.

14. B. During, D. Matthes, and G. Toscani, Kinetic equations modelling wealth redistribution: a comparison of approaches, Physical Review E, vol. 78, p. 056103, 2008. 


\section{Dolfin}

15. B. During, D. Matthes, and G. Toscani, A Boltzmann-type approach to the formation of wealth distribution curves, Rivista di Matematica della Universitá di Parma, vol. 8, pp. 199-261, 2009.

16. D. Acemoglu and J. A. Robinson, Economic backwardness in political perspectives, American Political Science Review, vol. 100, pp. 115-131, 2006.

17. L. Robbins, An Essay on the Nature and Significance of Economic Science (1932). Macmillian and Co., 2013.

18. D. Acemoglu and J. A. Robinson, Political losers as a barrier to economic development, American Economic Review, vol. 90, pp. 126-130, 2000.

19. L. Leonida, D. M. A. Patti, and P. Navarra, The political replacement effect: a panel data analysis, Oxford Bulletin of Economics and Statistics, vol. 75, pp. 785-805, 2013.

20. P. Navarra, Addressing quantification of "turbulence" in the "political replacement effect": Comment on "modeling human behavior in economics and social science" by M. Dolfin, L. Leonida \& N. Outada, Physics of Life Reviews, vol. 22-23, pp. 46-47, 2017. 